\title{
HOMOMORPHISMS OF CONTINUOUS PSEUDOGROUPS
}

\author{
JOSEPH E. D'ATRI*
}

Introduction. In this paper we attempt to set up a notion of homomorphism for continuous pseudogroups and show that the kernel exists (as a continuous pseudogroup) in the transitive case. This paper is really an extension of the paper by Kuranishi and Rodrigues [11] which essentially examines the question of the existence (as a continuous pseudogroup) of an image of a homomorphism. A certain amount of overlap in definitions and statements of results was unavoidable, especially in sections 2 and 3, but for many proofs and constructions the reader is referred to that paper. For the basic notions of the theory of continuous pseudogroups as used in section 4, see Kuranishi [9] and for the terminology of the Cartan-Kähler theory used in section 5, see Kuranishi [6]. Fuller expositions may be found in Cartan [1], Kähler [4], Kumpera [5], Kuranishi [7], and Schouten and v.d. Kulk [13].

The author would like to take this opportunity to express his deep gratitude to Prof. M. Kuranishi for his assistance in this work for the last several years.

\section{$\S 1$. Basic Notions}

Since important parts of this theory hold only in the real analytic case, we assume that all manifolds, maps, etc. are real analytic. Most frequently, we assume that our manifolds are pointed manifolods, i.e., pairs consisting of a manifold and a distinguished point in that manifold. We write $(M, p)$ to indicate that $p$ is the distinguished point of $M$. Since our theory is primarily local, it will often be desirable to "shrink" $M$, i.e., to replace $M$ by an open, connected neighborhood of $p$. We will usually not indicate such shrinkings in our notation. It is usually assumed that a mapping from one manifold to

Received February 21, 1964.

*) This paper is based on part of the author's Ph. D. dissertation at Princeton University which was written while the author was partially sponsored by a project of the U.S. Army Research Office. 
another will carry the distinguished point into the distinguished point.

We assume familiarity with Ehresman's concept of jet spaces. If $M$ is a manifold, we let $J^{k}(M)$ denote the space of invertible $k$-jets on $M$, while $\alpha$ and $\beta$ will denote respectively the source and target projections. We also let $A^{k}(M)$ denote the space of analytic $k$-forms on $M$ considered as a module over $A^{\circ}(M)$, which is just the space of functions on $M$.

Given manifolds $M$ and $N$ and a map $\rho$ from $M$ onto $N$, we will call the triple $(M, N, \sigma)$ a fibered manifold if $\rho_{*}$ maps the tangent space to $M$ at each point onto the tangent space to $N$ at the image point. We usually assume that each fiber is connected. Note that if we let $I^{k}(\not)$ denote the identity $k$-jet on $M$ at $p$, then $\left(J^{k}(M), I^{k}(p)\right)$ is a pointed manifold and $\left(J^{k}(M), M, \alpha\right)$ and $\left(J^{k}(M)\right.$, $M, \beta)$ are fibered manifolds. If $(M, N, \rho)$ is a fibered manifold and we are given a point $p \in M$ and a coordinate system $(x)=\left(x_{i}\right)$ defined in a neighborhood of $\rho(p)$, it is always possible to choose coordinates $(y)=\left(y_{j}\right)$ defined on a neighborhood of $p$ such that $x_{j} \circ \rho=y_{j}$. Such coordinates $(y)$ will be called fibering coordinates and we will loosely refer to this process as raising the coordinates $(x)$ to $p$. We will usually not distinguish notationally between $x_{i} \circ \rho$ and $x_{i}$. If we are given a "diagram" of manifolds and maps, we will call this a diagram of fibered manifolds if each "arrow" $M \underset{\rho}{\longrightarrow} N$ gives a fibered manifold structure $(M, N, \rho)$. Lastly, if it is necessary to shrink any of the manifolds in such a diagram, it will be assumed that the others are also shrunk so as to preserve the fibered manifold structures.

By a homeomorphism element on a manifold $M$, we will mean a homeomorphism $f$ from an open set of $M$ (frequently denoted by $U(f)$ ) onto another open set of $M$ (frequently denoted by $V(f)$ ) which is bianalytic. If $f$ and $g$ are homeomorphism elements on $M$ such that $V(g) \cap U(f)$ is not empty, then we define $f \circ g$ in the obvious way as a homeomorphism element with domain equal to $g^{-1}(U(f))$. Let $\Gamma$ be a set of homeomorphism elements on $M$. Then $\Gamma$ is called a pseudogroup of transformations on $M$ if : (1) $f$ contained in $\Gamma$ implies that $f^{-1}$ is contained in $\Gamma$, and $(2) f$ and $g$ contained in $\Gamma$ implies that $f \circ g$ is contained in $\Gamma$ whenever defined. Let $\bar{\Gamma}$ denote the collection of all homeomorphism elements $f$ on $M$ such that for any point $x \in U(f)$, there exists an element $g$ in $\Gamma$ which coincides with $f$ in some neighborhood of $x, \quad \bar{T}$ is again a pseudogroup which is called the completion of $\Gamma . \quad \Gamma$ itself is called 
complete if $\Gamma=\bar{T}$. We call $\Gamma$ transitive if for any two points $x$ and $y$ in $M$, there exists an element $g$ in $\Gamma$ such that $g(x)=y$. If $M_{1}$ is an open submanifold of $M$, then by $\Gamma \mid M_{1}$ we will mean the set of $g \in \Gamma$ such that $U(g)$ and $V(g)$ are contained in $M_{1}$. Every time that the manifold $M$ is shrunk, it will be assumed that $\Gamma$ is also shrunk in this way.

An important class of pseudogroups is formed as follows. Let $A_{R}^{1}(M)$ denote the space of analytic Pfaffians on $M$ considered as a vector space over the reals and let $\Omega$ be any subspace of $\Lambda_{R}^{2}(M)$. Then $\Gamma(\Omega)$ will denote the set of all homeomorphism elements $f$ on $M$ such that $f^{*} \omega=\omega$ on $U(f)$ for any $\omega \in \Omega$. Clearly $\Gamma(\Omega)$ is a complete pseudogroup on $\mathcal{M}$.

\section{§. Cartan Spaces}

Definition 2.1. Let $(M, p)$ be a pointed manifold and suppose we have a system of real analytic Pfaffians $\omega_{1}, \ldots, \omega_{n}, \widetilde{\omega}_{1}, \ldots, \widetilde{\omega}_{m}$ on $M$ such that:

(1) $\omega_{1}, \ldots, \omega_{n}, \widetilde{\omega}_{1}, \ldots, \widetilde{\omega}_{m}$ are linearly independent at each point

(2) We can write $d \omega_{i}=c_{i}^{j k} \omega_{j} \wedge \omega_{k}+a_{i}^{j \lambda} \omega_{j} \wedge \widetilde{\omega}_{\lambda}$ where the $c_{i}^{j k}$ and $a_{i}^{j \lambda}$ are constants.

By the structure matrices of such a system, we mean the matrices $a^{\lambda}=\left(a_{i}^{j \lambda}\right)$. If such a system also satisfies :

(3) The matrices $a^{1}, \ldots, a^{m}$ are linearly independent then $\left(\omega_{1}, \ldots, \omega_{n} ; \widetilde{\omega}_{1}, \ldots, \widetilde{\omega}_{m}\right)$ is a Cartan basis. The $\widetilde{\omega}_{\lambda}$ will be called supplementary forms for the $\omega_{i}$ and the equation in (2) will be called the structure equation for the basis. It will usually be assumed that the $c_{i}^{j k}$ have been made skew-symmetric in the upper indices so that the structure equation is uniquely determined. We remark that if we have a system of forms $\left(\omega_{i} ; \eta_{\sigma}\right)$ satisfying (1) and (2), then we can find forms $\widetilde{\omega}_{\lambda}$ in the real vector space generated by the $\eta_{s}$ so that $\left(\omega_{i} ; \widetilde{\omega}_{\lambda}\right)$ is a Cartan basis.

Now suppose we have vector subspaces $\Omega$ and $\Pi$ of $\Lambda_{R}^{1}(M)$ and suppose we can find a Cartan basis $\left(\omega_{1}, \ldots, \omega_{n} ; \widetilde{\omega}_{1}, \ldots, \widetilde{\omega}_{m}\right)$ such that $\left(\omega_{1}, \ldots, \omega_{n}\right)$ is a basis for $\Omega$ and $\left(\omega_{1}, \ldots, \omega_{n}, \widetilde{\omega}_{1}, \ldots, \widetilde{\omega}_{m}\right)$ is a basis for $I$. Then $(\Omega, \Pi)$ is called a Cartan system and $\left(\omega_{i} ; \widetilde{\omega}_{\lambda}\right)^{\prime}$ is called a Cartan basis for $(\Omega, \Pi)$. It is easy to see that if $(\Omega, \Pi)$ is a Cartan system, then $\left(\omega_{i} ; \widetilde{\omega}_{\lambda}\right)$ is a Cartan basis for $(\Omega, \Pi)$ if and only if $\left(\omega_{i}\right)$ is a basis for $\Omega$ and $\left(\omega_{i}, \widetilde{\omega}_{\lambda}\right)$ is a basis for II. The system is called complete if $\operatorname{dim} \Pi=\operatorname{dim} M$. 
Lastly suppose we have any subspace $\Omega$ of $\Lambda_{R}^{1}(M)$ for which there exists a subspace $\Pi$ making $(\Omega, \Pi)$ into a Cartan system. Then we call $\Omega$ a Cartan space and $(\Omega, \Pi)$ will be called a system for $\Omega$.

Definition 2.2. Let $(\Omega, \Pi)$ be a Cartan system with basis $\left(\omega_{i} ; \widetilde{\omega}_{\lambda}\right)$ and structure matrices $\left(a_{i}^{j \lambda}\right)$. Let $\Omega^{*}$ denote the dual of $\Omega$ with dual basis $\omega^{1}, \ldots$, $\omega^{n}$ and let $a^{\lambda} \in \operatorname{Hom}\left(\Omega^{*}, \Omega^{*}\right)$ be defined by $a^{\lambda}\left(\omega^{j}\right)=a_{i}^{j \lambda} \omega^{i}$. The $a^{\lambda}$ generate a subspace of $\operatorname{Hom}\left(\Omega^{*}, \Omega^{*}\right)$ which is independent of the choice of Cartan basis. This subspace is denoted by $L(\Omega, \Pi)$ or simply $L$. Choosing a Cartan basis induces natural bases on $\Omega^{*}$ and $L$ and hence associates to every element $b \in$ $\operatorname{Hom}\left(\Omega^{*}, L\right)$ a canonical matrix representation $\left(b_{\lambda}^{j}\right)$. We let $\mathfrak{D}(\Omega, \Pi)$ denote the subspace of elements $b$ satisfying

$$
a_{i}^{j \lambda} b_{\lambda}^{k}-a_{i}^{k \lambda} b_{\lambda}^{j}=0 \text { for all } i, j \text {, and } k \text {. }
$$

This is called the derived space in Kuranishi-Rodrigues [11] and the prolonged space in Guillemin-Sternberg [3]. This space is also independent of the choice of Cartan basis. Finally the system $(\Omega, \Pi)$ will be called involutive if $L(\Omega$, $I I)$ is involutive in the sense defined in Kuranishi-Rodrigues.

Proposition 2.1. Let $(\Omega, \Pi)$ and $\left(\Omega, \Pi^{\prime}\right)$ be systems for the Cartan space s. Then $\operatorname{dim} \Pi=\operatorname{dim} \Pi^{\prime}, \Pi_{q}=\Pi_{q}^{\prime}$ for any point $q$, and $L(\Omega, \Pi)=L\left(\Omega, \Pi^{\prime}\right)$. Now let $\left(\omega_{i} ; \widetilde{\omega}_{\lambda}\right)$ be a Cartan basis for $(\Omega, \Pi)$ with structure matrices $\left(a_{i}^{j \lambda}\right)$. Then there exists a basis of $\left(\Omega, \Pi^{\prime}\right)$ of the form $\left(\omega_{i} ; \xi_{\lambda}\right)$ satisfying:

(1) $\widetilde{\omega}_{\lambda}=\xi_{\lambda}+h_{\lambda}^{k} \omega_{k}$ where $h_{\lambda}^{k}$ are functions on $M$.

(2) For each point $q$, the matrix $\left(h_{\lambda}^{k}(q)\right)$ is the canonical representation, with respect to the bases induced by $\left(\omega_{i} ; \widetilde{\omega}_{\lambda}\right)$, of an element of $\mathscr{D}(\Omega, \Pi)$.

(3) The structure constants of the two bases are the same.

Conversely, if $\xi_{1}, \ldots, \xi_{m}$ are Pfaffians on $M$ satisfying (1) and (2), then $\left(\omega_{i} ; \hat{\zeta}_{\lambda}\right)$ is a basis of a system for $\Omega$ and (3) follows. We remark that we can always choose the supplementary forms $\xi_{\lambda}$ so that $h_{\lambda}^{k}(p)=0$ where $p$ is the distinguished point of $M$.

See Kuranishi Rodriques for proof.

Because of this proposition, we can introduce the notation $L(\Omega)$ and $\mathscr{D}(\Omega)$ to denote the spaces of Definition 2.2. In particular we see that the property of being involutive is a property of $\Omega$ alone and is not dependent on the choice of system. The same holds true for completeness. 
Definition 2.3. A vector subspace $\Omega^{\prime}$ of a Cartan space $\Omega$ is a Cartan subspace-if $\Omega^{\prime}$ itself is a Cartan space.

Proposition 2.2. A vector subspace $\Omega^{\prime}$ of a Cartan space $\Omega$ is a Cartan subspace if and only if the equation $\Omega^{\prime}=0$ is completely integrable. Under these conditons, if $(\Omega, \Pi)$ is a system for $\Omega$, then there is a unique system $\left(\Omega^{\prime}, \Pi^{\prime}\right)$ of $\Omega^{\prime}$ such that $\Pi^{\prime} \subset \Pi$. The vector space $\Gamma^{\prime} \cap \Omega$ is independent of the choice of system $(\Omega, \Pi)$.

Proof. The first assertion appears in Kuranishi-Rodrigues and their proof shows that under these conditions, $\Omega^{\prime}$ will have a system $\left(\Omega^{\prime}, \Pi^{\prime}\right)$ with $\Gamma^{\prime} \subset \Pi$. Suppose we had another system $\left(\Omega^{\prime}, \Pi^{\prime \prime}\right)$ with $\Pi^{\prime \prime} \subset \Pi$. Then at any point $q$, $\Pi_{q}^{\prime}=\Pi_{a}^{\prime \prime}$. Since any two subspaces of $\Pi$ which agree at each point must be equal, we have $I^{\prime}=\Pi^{\prime \prime}$.

Now let $(\Omega, \Xi)$ be another system for $\Omega$ and let $\left(\Omega^{\prime}, \Xi^{\prime}\right)$ be the unique system for $\Omega$ : induced by $\Xi$. We can choose the basis $\left(\omega_{i} ; \widetilde{u}_{\lambda}\right)$ of $(\Omega, \Pi)$ so that

$$
\left(\omega_{1}, \ldots, \omega_{n^{\prime}} ; \omega_{n^{\prime}+1}, \ldots, \omega_{n^{\prime}+s^{\prime}}, \widetilde{\omega}_{1}, \ldots, \widetilde{\omega}_{m^{\prime}-s^{\prime}}\right)
$$

is a basis for $\left(\Omega^{\prime}, \Pi^{\prime}\right)$. We can also choose a basis $\left(\omega_{i} ; \xi_{\lambda}\right)$ of $(\Omega, \Xi)$ so that the conclusions of Proposition 2.1 hold, in particular so that $\left(\omega_{i} ; \widetilde{\omega}_{\lambda}\right)$ and $\left(\omega_{i}\right.$; $\left.\xi_{\lambda}\right)$ have the same structure constants. Then we see easily that

$$
\left(\omega_{1}, \ldots, \omega_{n^{\prime}} ; \omega_{n^{\prime}+1}, \ldots, \omega_{n^{\prime}+s^{\prime}}, \xi_{1}, \ldots, \xi_{m^{\prime}-s^{\prime}}\right)
$$

is a Cartan basis, having the same structure constants as (1), and hence, by uniqueness, must be a basis for $\left(\Omega^{\prime}, \Xi^{\prime}\right)$. Clearly then $\Pi^{\prime} \cap \Omega=\Xi^{\prime} \cap \Omega$.

Definition 2.4. Let $\Omega^{\prime}$ be a Cartan subspace of the Cartan space $\Omega$. Let $(\Omega, I)$ be a system for $\Omega$ and let $\left(\Omega^{\prime}, \Pi^{\prime}\right)$ be the induced system. Then $s(\Omega$, $\left.\Omega^{\prime}\right)=\operatorname{dim}\left(\Pi^{\prime} \cap \Omega\right)-\operatorname{dim} \Omega^{\prime}$ is called the supplementary dimension of $\Omega^{\prime}$ in $\Omega$.

Definition 2.5. Let $(M, N, \rho)$ be a fibered manifold and let $\Omega$ and $\Omega^{\prime}$ be Cartan spaces on $M$ and $N$ respectively. The Cartan spaces are called compatible if $\rho^{*} \Omega^{\prime} \subset \Omega$. In this case $\rho^{*} \Omega^{\prime}$ is a Cartan subsystem and we set $s\left(\Omega, \Omega^{\prime}\right)=s(\Omega$, $\left.\rho^{*} \Omega^{\prime}\right)$.

Definition 2.6. Suppose we are given a diagram of fibered manifolds. This is called a diagram of fibered manifolds and Cartan spaces if a complete 
Cartan space is given on each manifold and if these are all compatible with the fibrations.

Definition 2.7. Fix a complete Cartan space $\Omega$ on a pointed manifold ( $M$, $p)$, a system $(\Omega, \Pi)$, and a basis $\left(\omega_{1}, \ldots \omega_{n} ; \widetilde{\omega}_{1}, \ldots, \widetilde{\omega}_{m}\right)$ with structure matrices $\left(a_{i}^{j \lambda}\right)$. Let $D=M \times M$ and let $\alpha$ and $\beta$ denote the projections of $D$ onto the first and second factors respectively. Set $\Omega_{i}=\beta^{*} \omega_{i}$ and $\Pi_{\lambda}=\beta^{*} \widetilde{\omega}_{\lambda}$. We will also want to consider $\alpha^{*} \omega_{i}$ and $\alpha^{*} \widetilde{\omega}_{\lambda}$ but we will simply write these as $\omega_{i}$ and $\widetilde{\omega}_{\lambda}$.

Let $\Sigma(\Omega)$ be the exterior differential system on $D$ generated as an ideal by $\omega_{i}-\Omega_{i}$ and $d \omega_{i}-d \Omega_{i}$. Let $\Theta(\Omega)=\alpha^{*}\left(\Lambda^{1}(M)\right)$, i.e., $\Theta$ is equal to the $\Lambda^{0}(D)$ submodule of $\Lambda^{1}(D)$ generated by $\omega_{i}$ and $\widetilde{\omega}_{\lambda} . \quad \Theta$ is a system of $n+m$ independent variables. The pair $(\Sigma, \Theta)$ is called the exterior differential system with independent variables assosicated to the Cartan space $\Omega$. It is clearly independent of the choice of basis or system. Using the structure equations for $\left(\omega_{i} ; \widetilde{\omega}_{\lambda}\right)$, we see that $\Sigma$ can also be characterized as the ideal generated by $\dot{\omega}_{i}-\Omega_{i}$ and $\boldsymbol{a}_{i}^{j \lambda}\left(\omega_{j} \wedge\left(\widetilde{\omega}_{\lambda}-\Pi_{\lambda}\right)\right)$.

Let $S \subset D$. Then $S$ is a solution manifold of $(\Sigma, \Theta)$ if and only if $S$ is the graph of a transformation in $\Gamma(\Omega)$. Also it is easy to show that the set of $n+m$ dimensional integral elements of $(\Sigma(\Omega), \Theta(\Omega))$ is a submanifold of the $n+m$ dimensional Grassmannian of $M$ with dimension equal to $2 \operatorname{dim}(D)+$ $\operatorname{dim} \mathfrak{D}(\Omega)$. Finally it is well known that the Cartan space is involutive if and only if the associated exterior differential system is involutive in the sense of the Cartan-Kähler theory.

\section{§ 3. Prolongations of Cartan Spaces}

In this section we fix a complete Cartan space $\Omega$ on a pointed manifold $(M, p)$. We let $J^{k}(M, p)$ denote the manifold of invertible $k$-jets with target $p$. For any $X=j_{x}^{1} f \in J^{1}(M, p)$ and any form $\theta$ on $M$, we note that $\left(f^{*} \theta\right)_{x}$ is independent of the choice of representative $f$. Let $X^{*} \theta=\left(f^{*} \theta\right)_{x}$.

Definition 3.1. Let $B(\Omega, M, p)$, written $B(\Omega)$ for short, be equal to the set of $X \in J^{\mathrm{i}}(M, p)$ such that $X^{*} \omega=\omega$ and $X^{*} d \omega=d \omega$ for all $\omega \in \Omega$.

Choose a system $(\Omega, \Pi)$ and a basis $\left(\omega_{1}, \ldots, \omega_{n} ; \widetilde{\omega}_{1}, \ldots, \widetilde{\omega}_{m}\right)$. Let $\left(x_{r}\right)$ be a coordinate system on $M$ with origin at $p$. We now construct a coordinate system on $J^{1}(M, p)$ associated to these choices. For any $X \in J^{1}(M, p)$, set 
$x_{r}(X)=x_{r}(\alpha(X))$. Also we can uniquely define $p_{i}^{r}(X), q_{\lambda}^{r}(X)$ so that

$$
\begin{aligned}
& X^{*} \omega_{i}=p_{i}^{j}(X)\left(\omega_{j}\right)_{x}+p_{i}^{n+\mu}(X)\left(\widetilde{\omega}_{\lambda}\right)_{x} \\
& X^{*} \widetilde{\omega}_{\lambda}=q_{\lambda}^{j}(X)\left(\omega_{j}\right)_{x}+q_{\lambda}^{n+\mu}(X)\left(\widetilde{\omega}_{\lambda}\right)_{x}
\end{aligned}
$$

where $x=\alpha(X)$. It is clear that $\left(x_{r}, p_{i}^{r}, q_{\lambda}^{r}\right)$ give coordinates on $J^{1}(M, p)$. We find that $B(\Omega)$ is defined as a subvariety of $J^{1}(M, p)$ by the equations

$$
\begin{aligned}
& p_{i}^{j}(X)=\delta_{i}^{j}, \quad p_{i}^{n+\mu}(X)=0 \\
& a_{i}^{j \lambda} q_{\lambda}^{k}(X)-a_{i}^{k \lambda} q_{\lambda}^{j}(X)=0 \\
& q_{\lambda}^{n+\mu}(X)=\delta_{\lambda}^{\mu} .
\end{aligned}
$$

Let $b^{s}, \sigma=1, \ldots, \delta$ be a basis of $\mathscr{D}(\Omega)$ and let $\left(b_{\lambda}^{i s}\right)$ be the matrix representation of $b^{\sigma}$ associated by the choice of Cartan basis. From (2), we know that if $X \in B(\Omega)$, then $\left(g_{\lambda}^{i}(X)\right)$ represents an element $g(X)$ in $\mathscr{D}(\Omega)$ and so we can write $q(X)=b^{\top} u_{s}(X)$, i.e., $q_{\lambda}^{i}(X)=b_{\lambda}^{i \sigma} u_{\sigma}(X)$. It is clear that $\left(x_{r}, u_{\sigma}\right)$ form coordinates on $B(\Omega)$ which is therefore a submanifold. $I^{1}(p)$ is taken as the distinguished point of $B(\Omega)$. Note also that $(B(\Omega), M, \alpha)$ is a fibered manifold, in fact the fibration by maximal integral elements of the system $d u_{s}=0$

Now define Pfaffians $\omega_{n+\lambda}, 1 \leq \lambda \leq m$, on $B(\Omega)$ by the equation $\left(\omega_{n+\lambda}\right)_{x}=$ $X^{*} \widetilde{\omega}_{\lambda}$. The real vector space generated by $\omega_{1}, \ldots, \omega_{n+m}$ is independent of the choice of basis or system of $\Omega$. This vector space is denoted by $P(\Omega)$. Kuranishi and Rodrigues show that $\Gamma(P(\Omega))$ is equal to the set of homeomorphism elements on $B(\Omega)$ which coincide locally with a $P f$ for some $f \in \Gamma(\Omega)$. Here $P f$ is the homeomorphism element on $J^{1}(M, p)$ defined in $\alpha^{-1}(U(f))$ by the formula

$$
P f(X)=X \circ\left(j_{x}^{1} f\right)^{-1}
$$

where $x=\alpha(X)$. If $f \in L(\Omega)$, then it is clear that $P f$ induces a homeomorphism element, also denoted $P f$, on $B(\Omega)$.

Let $\left(M, M^{\prime}, \rho\right)$ be a fibered manifold with $\operatorname{dim} M=d, \operatorname{dim} M^{\prime}=d^{\prime}$ and suppose we have compatible, complete Cartan spaces $\Omega$ and $\Omega^{\prime}$ on $M$ and $M^{\prime}$ respectively. Let $\left(y_{t}\right)$ be a system of coordinates on $M^{\prime}$ and raise these to coordinates $\left(x_{s}\right)$ on $M$. For any $X=j_{x}^{1} f \in J^{1}(M, p)$, let

$$
V_{s}^{n}=\left.\frac{\partial f^{o}}{\partial x_{s}}\right|_{x}
$$

and similarly for any $Y=j_{y}^{1} g \in J^{1}\left(M^{\prime}, p^{\prime}\right)$, define $w_{i}^{\tau}$. Then $\left(x_{s}, v_{s}^{\tau}\right)$ and $\left(y_{t}\right.$, 
$\left.w_{t}^{\tau}\right)$ give coordinates on $J^{1}(M, p)$ and $J^{1}\left(M^{\prime}, p^{\prime}\right)$ respectively. For any $X \in B(\Omega)$, define $\rho^{k}(X)=Y \in \dot{J}^{1}\left(M^{\prime}, p^{\prime}\right)$ by the equations

$$
\begin{aligned}
& \alpha(Y)=\rho(\alpha(X)) \\
& w_{t}^{\tau}(Y)=v_{t}^{\tau}(X) \text { for } \tau, t=1, \ldots, d^{\prime} .
\end{aligned}
$$

A simple calculation shows that $Y \in B\left(\Omega^{\prime}\right)$.

A priori it would appear that the map $\rho^{B}$ depends on the choice of coordinates. However if

$$
v_{s}^{\sigma}(X)=0 \text { for } 1 \leq \sigma \leq d^{\prime} \text { and } d^{\prime}<s \leq d
$$

then it is easy to see that $\rho^{B}(X)$ is independent of choice of coordinate. One can show that (3) holds for all $X \in B(\Omega)$ and so $\rho^{B}: B(\Omega) \rightarrow B\left(\Omega^{\prime}\right)$ is well defined. In addition, $\left(\rho^{B}\right)^{*}\left(P\left(\Omega^{\prime}\right)\right) \subset P(\Omega)$. This calculation also shows that for $f \in \Gamma(\Omega)$ and $x \in U(f)$, there is a unique $g \in \Gamma\left(\Omega^{\prime}\right)$, defined in a neighborhood of $y=\rho(x)$, such that $\rho \circ f=g \circ \rho$ in a neighborhood of $x$. If $X \in B(\Omega)$ is of the form $j_{x}^{1} f$ where $f \in \Gamma(\Omega)$, we find that $\rho^{B}(X)=j_{y}^{1} g$. This makes the description of $\rho^{B}$ particularly simple when $\Omega$ is involutive since then every element of $B(\Omega)$ has this form.

It should be noted here that the operator $B$ is actually a functor from the category of pointed manifolds with complete Cartan spaces and compatible fibrations to the category of pointed manifolds and ordinary maps. The following propositions, from Kuranishi-Rodrigues, show the effect of the operator $P$ when $\Omega$ is involutive.

Proposition 3.1. If $\Omega$ is involutive then $P(\Omega)$ is a complete, involutive Cartan space on $B(\Omega)$.

Proposition 3.2. Let $\left(M, M^{\prime}, \rho\right)$ be a fibered manifold with complete, compatible Cartan spaces $\Omega$ and $\Omega^{\prime}$ and suppose $\Omega$ is involutive. Then $P\left(\Omega^{\prime}\right) \mid \rho^{B}(B(\Omega)$ is a complete Cartan space compatible with $P(\Omega)$ under the fibration $(B(\Omega)$, $\left.\rho^{\prime \prime}(B(\Omega)), \rho^{B}\right)$. Also each map in $\Gamma\left(P\left(\Omega^{\prime}\right) \mid \rho^{\prime \prime}(B(\Omega))\right.$ is the restriction of a map in $I^{\prime}\left(P\left(\Omega^{\prime}\right)\right)$ and conversely.

This section concludes with a lemma needed in our main proof.

Lemma 3.3. Suppose we have complete Cartan spaces $\Omega$ and $\Omega^{\prime}$ on $(M, p)$ and $\left(M, f^{\prime}\right)$ respectively and a compatible fibered manifold $\left(M, M^{\prime}, \kappa\right)$. Assume 
that $\Omega$ is involutive and $S\left(\Omega, \Omega^{\prime}\right)=0$. Let $M_{1}$ equal $B(\Omega, M, p)$ and $M_{1}^{\prime} \subset B\left(\Omega^{\prime}\right.$, $\left.M^{\prime}, p^{\prime}\right)$ equal the image of $M_{1}$ by $\kappa^{R}$. Then $\left.S\left(P(\Omega), P\left(\Omega^{\prime}\right) \mid M_{1}^{\prime}\right)\right)=0$

Proof. Let $(\Omega, \Pi)$, respectively $\left(\Omega^{\prime}, \Pi^{\prime}\right)$, be a system for $\Omega$, respectively $\Omega^{\prime}$. Let $\Omega^{*}=\kappa^{*} \Omega^{\prime}$ and let $\left(\Omega^{*}, \Pi^{*}\right)$ be the unique system with $\Pi^{*} \subset \Pi$. If $\theta$ is a Pfaffian on $M^{\prime}$, we let $\theta^{*}=\kappa^{*} \theta$. We can find bases

$$
\begin{aligned}
& \left(\omega_{1}, \ldots, \omega_{n} ; \widetilde{\omega}_{1}, \ldots, \widetilde{\omega}_{m}\right) \text { of }(\Omega, \Pi) \\
& \left(\theta_{1}^{*}, \ldots, \theta_{n^{\prime}}^{*} ; \xi_{1}, \ldots, \xi_{m^{\prime}}\right) \text { of }\left(\Omega^{*}, \Pi^{*}\right) \\
& \left(\theta_{1}, \ldots, \theta_{n^{\prime}} ; \eta_{1}, \ldots, \eta_{m^{\prime}}\right) \text { of }\left(\Omega^{\prime}, \Pi^{\prime}\right)
\end{aligned}
$$

so that $\eta_{\lambda}^{*}=\xi_{\lambda}-h_{\lambda}^{i} \theta_{i}^{*} ; \omega_{i}=\theta_{i}^{*}, 1 \leq i \leq n^{\prime} ; \widetilde{\omega}_{\lambda}=\xi_{\lambda}, 1 \leq \lambda \leq m^{\prime}$, where the $h_{\lambda}^{i}$ are functions on $M$ such that the matrix $\left(h_{\lambda}^{i}\right)$ represents an element $h$ in $\mathscr{D}\left(\Omega^{\prime}\right)$ at each point. We can assume that $h_{\lambda}^{i}(p)=0$. For simplicity, set $\Omega_{1}=P(\Omega)$ and $\Omega_{1}^{\prime}=P\left(\Omega^{\prime}\right) \mid M_{1}^{\prime}$. Lastly, choose a coordinate system $\left(x_{r}\right)$ on $M^{\prime}$ and lift this to a coordinate system $\left(x_{r}, y_{s}\right)$ on $M$.

Let $a_{j^{\prime}}^{j_{\mu}}$, respectively $\bar{a}_{i^{\prime}}^{i \lambda}$, be the structure constants of $\left(\omega_{j} ; \widehat{\omega}_{\mu}\right)$, respectively $\left(\theta_{i}^{*} ; \xi_{\lambda}\right)$ (and hence also of $\left.\left(\theta_{i} ; \eta_{\lambda}\right)\right)$. It is easy to see that

$$
\begin{aligned}
& a_{i^{\prime}}^{i \lambda}=\bar{a}_{i^{\prime}}^{i \lambda} \text { for } i, i^{\prime}=1, \ldots, n^{\prime} ; \lambda=1, \ldots, m^{\prime} \\
& a_{j^{\prime}}^{j u}=0 \text { for } j^{\prime} \leq n^{\prime} \text { and either } j>n^{\prime} \text { or } \lambda>m^{\prime} .
\end{aligned}
$$

From this we see that if $b=\left(b_{\mu}^{j}\right) \in \mathscr{D}(\Omega)$, then

$$
\varphi(b)=\left(b_{\lambda}^{i}\right)_{\substack{1 \leq i \leq n^{\prime} \\ 1 \leq \lambda=m^{\prime}}}
$$

is in $D\left(\Omega^{\prime}\right)$ and the mapping $\varphi$ defined in this way is a vector space homomorphism. Let $D$ equal the image of $\mathscr{D}(\Omega)$ by $\varphi$ and let $\psi$ be an injection of $D$ into $\mathscr{D}(\Omega)$ so that $\varphi \circ \psi$ is the identity and $\mathscr{D}(\Omega)=\psi(D) \oplus \operatorname{Ker} \varphi$. Let $b^{1}, \ldots, b^{t}$ be a basis for $\operatorname{Ker} \varphi$.

An element $X$ in $B(\Omega)$ has a unique representation of the form $\left(x_{r}, y_{s}\right.$, $q(X))$ where $q(X)=\left(q_{\mu}^{j}(X)\right)$ is in $\mathfrak{D}(\Omega)$ and any expression of this form defines an element in $B(\Omega)$. Likewise any element in $B\left(\Omega^{\prime}\right)$ has a unique representation $\left(x_{r}, \bar{q}(X)\right)$ where $\bar{q}(X)=\left(\bar{q}_{\lambda}^{i}(X)\right)$ is in $\mathscr{D}\left(\Omega^{\prime}\right)$. Let $X$ be in $B(\Omega)$. Since $\Omega$ is involutive, there is a $g$ in $\Gamma(\Omega)$ with $j_{(x, y)}^{1} g=X$ and since $\kappa^{*}\left(\Omega^{\prime}\right) \subset \Omega, g$ in a neighborhood of $(x, y)$ is the prolongation of sorne $f$ in $\Gamma\left(\Omega^{\prime}\right)$. Then

$$
\left(f^{*} \eta_{\lambda}\right)_{x}=\eta_{\lambda}+\bar{q}_{\lambda}^{i}\left(\kappa^{B}(X)\right) \theta_{i} .
$$

Raising this equation to $(x, y)$ by $\dot{\kappa}^{*}$ and comparing with the equation for $g^{*} \widetilde{\omega}_{\lambda}$, 
we have

$$
\bar{q}_{\lambda}^{i}\left(\kappa^{B}(X)\right)=q_{\lambda}^{i}(X)-h_{\lambda}^{i}(x, y)
$$

or

$$
\bar{q} \circ \kappa^{B}=\varphi \circ g-h \circ \alpha .
$$

Now $\left(M_{1}^{\prime}, M_{1}, \alpha\right)$ is a fibered manifold so we can find coordinates $\left(x_{r}, u_{\sigma}\right)$ on $M_{1}^{\prime}$. Let $X \in M_{1}=B(\Omega)$. Then

$$
q(X)-\psi \circ\left(\bar{q} \circ \kappa^{K}+h \circ \alpha\right)(X)
$$

is in the kernel of $\varphi$ and so can uniquely be written as

$$
v_{1}(X) b^{1}+\cdots+v_{t}(X) b^{t}
$$

Since $\left(M_{1}, M_{1}^{\prime}, \kappa^{k}\right)$ is fibered manifold, it is easy to see that $\left(x_{r}, y_{s}, u_{\sigma}, v_{\tau}\right)$ give coordinates on $M_{1}$ compatible with the fibrations.

Let $\rho$ be a cross-section of $\left(M_{1}, M_{1}^{\prime}, \kappa^{R}\right)$ passing through $p_{1}=I(p)$. There exists a transformation family $\left\{g^{x}\right\}$ in $\Gamma\left(\Omega_{1}\right)$, indexed by $M_{1}$, so that $g^{x}(X)=p_{1}$ (although each $g^{x}$ need not be defined on all of $M_{1}$ ). We construct a similar transformation family $\left\{f^{\bar{x}}\right\}$ in $\Gamma\left(\Omega_{1}^{\prime}\right)$ by letting $f^{\bar{x}}$ be the local homeomorphism induced by $g^{\tau(\bar{X})}$ in a neighborhood of $\tau(\bar{X})$.

Supplementary forms $\widehat{\omega}_{2}^{1}$ for $\Omega_{1}$ are obtained by spreading around the $\left(d u_{\sigma}\right)_{p_{1}}$ and $\left(d v_{\tau}\right)_{p_{1}}$ using $\left\{g^{x}\right\}$ (this gives us a system $\left.\left(\Omega_{1}, \Pi_{1}\right)\right)$ and supplementary forms $\eta_{i,}^{1}$ for $\Omega_{1}^{\prime}$ are obtained by spreading around the $\left(d u_{\uparrow}\right)_{p_{1^{\prime}}}$ using $\left\{f^{\bar{x}}\right\}$ (this gives us a system $\left.\left(\Omega_{1}^{\prime}, \Pi_{1}^{\prime}\right)\right)$. Therefore $\left(\kappa^{B}\right)^{*} \eta_{\mu}^{1}$ is equal to $\widetilde{\omega}_{\mu}^{1}$ on the image of $\xi$ and hence surely at $p_{1}$. Let $\Omega_{1}^{*}=\left(\kappa^{k}\right)^{*} \Omega_{1}^{\prime}$ and let $\left(\Omega_{1}^{*}, \Pi_{1}^{*}\right)$ be the system with $\Pi_{1}^{*} \subset \Pi_{1}$. The supplementary forms $\xi_{\mu}^{1}$ for $\left(\Omega_{1}^{*}, \Pi_{1}^{*}\right)$ will be linear combinations with real cefficients of forms in $\Omega_{1}$ and of the $\widetilde{\omega}_{\lambda}^{1}$ and they can be chosen so that $\xi_{\mu}^{1}=\left(\kappa^{l}\right)^{*} \eta_{i \mu}^{1}$ at $p$. But therefore $\xi_{\mu}^{1}=\widetilde{\omega}_{\mu}^{1}$, i.e., $S\left(\Omega_{1}, \Omega_{1}^{\prime}\right)=0$.

\section{§4. Fibrations and equivalences}

Definition 4.1. Let $I$, respectively $I^{\prime \prime}$, be a complete pseudogroup on ( $M$, $p)$, respectively $\left(M^{\prime}, p^{\prime}\right)$, and suppose $\left(M, M^{\prime}, \kappa\right)$ is a fibered manifold. Then $(\Gamma, M, p) \stackrel{\kappa}{\longrightarrow}\left(\Gamma^{\prime}, M^{\prime}, p^{\prime}\right)$ is called a homomorphic fibration if for each $\dot{g} \in \Gamma$ and $y \in U(g)$, there is an $f \in \Gamma^{\prime}$ defined in a neighborhood of $\kappa(y)$ so that $\kappa \circ g=f \circ \kappa$ in a neighborhood of $y$. The fibration is called surjective, respectively injective, if in addition it satisfies condition $(S)$, respectively $(I)$, below. 
(S) There is an integer $k$ and for any $y \in M$, neighborhoods $U$ of $y$ and $\mathfrak{l l}$ of $I^{k}(\kappa(y))$ so that each transformation family in $\Gamma^{\prime}$ defined around a point $\kappa(z)$ where $z \in U$ and having $k$-jets in $\mathfrak{u}$ can be lifted to a covering family in $\Gamma$ defined around $z$, provided we restrict to small enough neighborhoods of $z$ and $\chi(z)$.

(I) There is an integer $k$ so that if $g \in \Gamma$ prolongs an $f \in \Gamma^{\prime}$ which satisfies $j_{x}^{k} f=I^{k}(x)$ for some $x \in U(f)$, then $g \mid \kappa^{-1}(x)$ is the identity.

The isomorphic fibrations of Kuranishi [9] are those which satisfy both (S) and $(I)$.

The kernel of a homomorphic fibration $(\Gamma, M, p) \stackrel{\kappa}{\rightarrow}\left(\Gamma^{\prime}, M^{\prime}, p^{\prime}\right)$, denoted by $\operatorname{Ker}(\kappa)$, is the complete pseudogroup of elements in $\Gamma$ which prolong indentities of $M^{\prime}$.

Definition 4.2. A pseudogroup $(\Gamma, M, p)$ is called an isomorphic prolongation of a pseudogroup $\left(\Gamma_{0}, M_{0}, p_{0}\right)$ if there exists an isomorphic fibration $(\Gamma$, $M, p) \rightarrow\left(\Gamma_{0}, M_{0}, p_{0}\right)$. A homomorphic fibration $(\Gamma, M, p) \rightarrow\left(\Gamma^{\prime}, M^{\prime}, p^{\prime}\right)$ is called an isomorphic prolongation of a homomorphic fibration $\left(\Gamma_{0}, M_{0}, p_{0}\right) \rightarrow\left(\Gamma_{0}^{\prime}, M_{0}^{\prime}\right.$, $\left.p_{0}^{\prime}\right)$ if there exist isomorphic fibrations making the following diagram commutative.

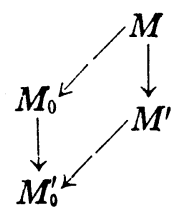

In the following definition, $J_{x}^{k}(M, x)$ denotes the Lie group of invertible $k$ jets on $M$ with source and target $x$ and $A_{x}^{k}(\Gamma, x)$ the abstract subgroup of $k$-jets coming from a pseudogroup $\Gamma$.

Definition 4.3. A complete pseudogroup $\Gamma$ on a manifold $(M, p)$ is called a continuous transformation pseudogroup if

(1) There is a fibered manifold $(M, N, \rho)$ whose fibers are the orbits of the action of $\Gamma$.

(2) Any $x \in M$ has a neighborhood $U$ and a transformation family $\left\{f^{y, z}\right\}$ in $\Gamma$ on $U$ with parameter manifold equal to $\left\{(y, z) \in L^{\top} \times U: \rho(y)=\rho(z)\right\}$ such that $f^{y, z}(y)=z$.

(3) For large enough $k, A_{x}^{k}(I ; x)$ is a Lie subgroup (in the sense of Chevalley) of $J_{x}^{k}(M, x)$ with dimension independent of $x$. 
(4) If $f$ is a homeomorphism element on $M$ and if $x$ is a point in $U(f)$ so that $j_{x}^{k} f \in A_{x}^{k}(\Gamma, x)$ for all $k$, then $f$, restricted to some neighborhood of $x$, is in $\Gamma$.

Condition (3) of this definition is slightly weaker than the corresponding condition of the definition in Kuranishi [9].

In Kuranishi [9], page 78, a sequence of standard isomorphic prolongations of a continuous transformation pseudogroup are defined. For example, the first standard prolongation of a Cartan pseudogroup $(\Gamma(\Omega), M, p)$ where $\Omega$ is complete and involutive is $(\Gamma(P(\Omega)), B(\Omega), I(p))$. It is easy to extend this definition to give a sequence of standard isomorphic prolongations of a homomorphic fibration of transitive continuous transformation pseudogroups.

Definition 4.4. Two continuous transformation pseudo roups are called equivalent if there exists a continuous transformation pseudogroup which is an isomorphic prolongation of each of them. Two homomorphic fibrations of continuous transformation pseudogroups are called equivalent if there exists a homomorphic fibration of continuous transformation pseudogroups which is an isomorphic prolongation of each of them.

Kuranishi [9] has shown that the first definition actually gives an equivalence relation. A slight extension of this proof shows the same for the second. An equivalence class of continuous transformation pseudogroups is called a continuous pseudogroup and a homomorphism of continuous pseudogroups is an equivalence class of fibrations.

If $(\Gamma, M, p) \stackrel{\kappa}{\rightarrow}\left(\Gamma^{\prime}, M^{\prime}, p^{\prime}\right)$ is a common isomorphic prolongation of $\left(\Gamma_{i}\right.$, $\left.M_{i}, p_{i}\right) \stackrel{\kappa i}{\rightarrow}\left(\Gamma_{i}^{\prime}, M_{i}^{\prime}, p_{i}^{\prime}\right), i=1,2$, then $\operatorname{Ker}(\kappa)$ is an isomorphic prolongation of each $\operatorname{Ker}\left(\kappa_{i}\right)$ even though none of these need be continuous. If each $\operatorname{Ker}\left(\kappa_{i}\right)$ is continuous then, though $\operatorname{Ker}(\kappa)$ may still not be continuous, there will exist some continuous common isomorphic prolongation of the $\operatorname{Ker}\left(\kappa_{i}\right)$ making them equivalent. Thus if at least one representative of a homomorphism $\varphi$ has a continuous kernel, we say that $\operatorname{Ker}(\varphi)$ exists and can unambiguously define it to be the equivalence class of the kernel of that representative.

Finally if a homomorphism $\varphi$ has an injective representative, then $\operatorname{Ker}(\varphi)$ exists and is trivial. The converse is not clear except in the case where $\varphi$ has a representative which is a fibration of transitive pseudogroups when it follows from the calculations of the next section. 


\section{§. The kernel problem}

In this section we prove our main result on the existence of kernels. Unfortunately our results are restricted to the transitive case. Quite likely a generalization of the theory of Cartan spaces, starting from a definition of Cartan basis where the structure "constants" could be functions (of the invariant variables) would overcome this restriction but we remark that the intransitive case has shown a peculiar obstinacy in the past. Thus Kuranishi's correspondence between infinite Lie $(F)$-groups and continuous pseudogroups and the results of Kuranishi and Rodrigues on the existence of homomorphic images of a given continuous pseudogroup must both be restricted to the transitive case

THEOREM 5.1. Let $\varphi$ be a homomorphism of continuous pseudogroups which has a representative $(\Gamma, M, p) \stackrel{\kappa}{\longrightarrow}\left(\Gamma^{\prime}, M^{\prime}, p^{\prime}\right)$ so that $\Gamma$ is transitive. Then $\varphi$ has a kernel.

Clearly $\Gamma^{\prime}$ will also be transitive and by the result of Kuranishi and Rodrigues, we can assume that $\kappa$ is surjective. By taking a high enough standard prolongation and using one of the main theorems of Kuranishi [9], we see that the representative can be chosen so that $I$, respectively $\Gamma^{\prime}$, is the Cartan pseudogroup determined by the complete, involutive Cartan space $\Omega$, respectively $\Omega^{\prime}$. It is obvious that every $g$ in $\Gamma=\Gamma(\Omega)$ leaves each element in $\kappa^{*} \Omega^{\prime}$ invariant. Hence, taking one more standard prolongation of $\Gamma$ if necessary, we may assume that $\kappa^{*} \Omega^{\prime} \subset \Omega$, i.e., that the Cartan spaces are compatible with the fibration. If in addition it could be assumed that $S\left(\Omega, \Omega^{\prime}\right)=0$, it turns out that the CartanKähler theory could be applied to $\Sigma(\Omega)$ and $\Sigma\left(\Omega^{\prime}\right)$ simultaneously in a very illuminating way. Thus our first task is to construct a representative for which this is so, starting from the representative we have at hand.

Since $\Omega$ is involutive, $P(\Omega)$ is a complete, involutive Cartan space on $B(\Omega)$ and we can define $B^{2}(\Omega)=B(P(\Omega), B(\Omega))$ and $P^{2}(\Omega)=P(P(\Omega))$. We have the same construction for $\Omega^{\prime}$. Since everything is involutive, it is easy to see that $\kappa^{B}(B(\Omega))=B\left(\Omega^{\prime}\right)$ and so we can define the map $\kappa^{2 B}=\left(\kappa^{B}\right)^{B}$ from $B^{2}(\Omega)$ to $B^{2}\left(\Omega^{\prime}\right)$. Continuing inductively, we can define the fibered manifold $\left(B^{s}(\Omega), B^{s}\left(\Omega^{\prime}\right), \kappa^{s l s}\right)$ bearing the compatible Cartan spaces $P^{s}(\Omega), P^{s}\left(\Omega^{\prime}\right)$ by the conditions

$$
\begin{aligned}
& B^{s}(\Omega)=B\left(P^{s-1}(\Omega), B^{s-1}(\Omega)\right) \\
& B^{s}\left(\Omega^{\prime}\right)=B\left(P^{s-1}\left(\Omega^{\prime}\right), B^{s-1}\left(\Omega^{\prime}\right)\right)
\end{aligned}
$$




$$
\kappa^{S B}=\left(\kappa^{(s-1) B}\right)^{B} .
$$

For simplicity, let $M_{s}=B^{s}(\Omega), M_{s}^{\prime}=B^{s}\left(\Omega^{\prime}\right), \Omega_{s}=P^{s}(\Omega), \Omega_{s}^{\prime}=P^{s}\left(\Omega^{\prime}\right)$ and $\kappa_{s}=\kappa^{s B}$. Kuranishi and Rodrigues have shown how to construct a complete Cartan space $\Omega^{4}$ on a pointed manifold $\left(M^{4}, p^{4}\right)$ and, for all sufficiently large $s$, maps making the following a commutative diagram of fibered manifolds and compatible Cartan spaces:

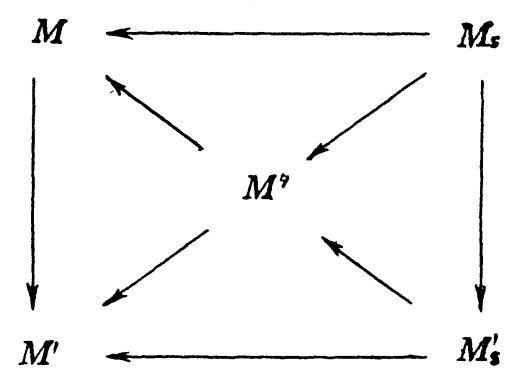

The point of their construction is that $S\left(\Omega, \Omega^{4}\right)=0$. In addition, one can easily show that in our situation, $(\Gamma(\Omega), M) \rightarrow\left(\Gamma\left(\Omega^{b}\right), M^{b}\right)$ is a surjective homomorphic fibration which is equivalent to $(\Gamma(\Omega), M, p) \rightarrow\left(\Gamma\left(\Omega^{\prime}\right), M^{\prime}, p^{\prime}\right)$ since $\left(\Gamma\left(\Omega^{b}\right), M^{b}\right)$ $\rightarrow\left(\Gamma\left(\Omega^{\prime}\right), M^{\prime}\right)$ is an isomorphic fibration.

However this still does not give us the representative of $\varphi$ which we want. The trouble is that $\Omega^{4}$ need not be involutive. To remedy this defect, consider the fibered manifold $\left(M, M^{A}, \kappa^{4}\right)$ with the compatible Cartan spaces $\Omega$ and $\Omega^{4}$ and let $\kappa_{1}^{\beta}$ be the natural map from $B(\Omega)=M_{1}$ into $B\left(\Omega^{\beta}\right)$. Let $M_{1}^{\beta}=\kappa_{1}^{\beta}\left(M_{1}\right)$ and let $\Omega_{1}^{\beta}=P\left(\Omega^{\beta}\right) \mid M_{1}^{\beta}$. Now consider the fibered manifold $\left(M_{1}, M_{1}^{\beta}, \kappa_{1}^{\xi}\right)$ with the compatible Cartan spaces $\Omega_{1}$ and $\Omega_{1}^{\xi}$ and let $\kappa_{2}^{\mathcal{y}}$ be the natural map from $B\left(\Omega_{1}\right)=M_{2}$ into $B\left(\Omega_{1}^{\xi}\right)$. Let the image of $\kappa_{2}^{\psi}$ be $M_{2}^{\xi}$ and let $\Omega_{2}^{\psi}=P\left(\Omega_{1}^{\psi}\right) \mid M_{2}^{\psi}$. We proceed inductively, constructing a sequence of fibered manifold structures $\left(M_{s}, M_{s}^{\xi}, \kappa_{s}^{\xi}\right)$ with compatible Cartan spaces $\Omega_{s}$ and $\Omega_{s}^{\mathcal{S}}$ which fit into the following commutative diagram of fibered manifolds and Cartan spaces.

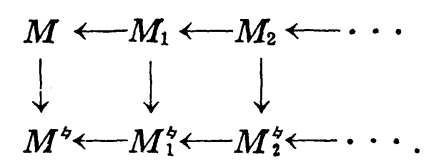

By Lemma 3.3, we see that $S\left(\Omega_{s}, \Omega_{s}^{\xi}\right)=0$. Also, the lower line of (4) is a Cartan sequence and so by Kuranisht-Rodrigues, $\Omega_{s}^{S}$ is involutive for large 
enough $s$. On the other hand, we can show by induction that for each $s$, we have the following commmutative diagram of fibered manifolds and compatible Cartan space :

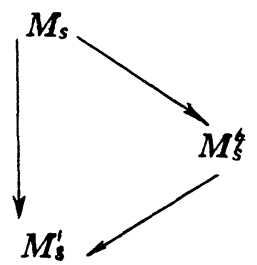

Finally, and again by induction, we see that $\left(\Gamma\left(\Omega_{s}\right), M_{s}\right) \rightarrow\left(\Gamma\left(\Omega_{s}^{\psi}\right), M_{s}^{\psi}\right)$ is a surjective homomorphic fibration which is equivalent to $\left(\Gamma\left(\Omega_{s}\right), M_{s}\right) \rightarrow\left(\Gamma\left(\Omega_{s}^{\prime}\right), M_{s}^{\prime}\right)$ since $\left(\Gamma\left(\Omega_{s}^{\xi}\right), M_{s}^{\xi}\right) \rightarrow\left(\Gamma\left(\Omega_{s}^{\prime}\right) M_{s}^{\prime}\right)$ is an isomorphic fibration.

Thus we know that we can find a representative of $\varphi$, which we might as well call $(\Gamma(\Omega), M) \rightarrow\left(\Gamma\left(\Omega^{\prime}\right), M^{\prime}\right)$ so that $\Omega, \Omega^{\prime}$ are complete, involutive Cartan spaces compatible with the fibration and $S\left(\Omega, \Omega^{\prime}\right)=0$. Let $(\Omega, \Pi)$, respectively $\left(\Omega, \Pi^{\prime}\right)$, be a system for $\Omega$, respectively $\Omega^{\prime}$. Let $\Omega^{*}=\kappa^{*} \Omega^{\prime}$ and let $\left(\Omega^{*}, \Pi^{*}\right)$ be the unique system with $\Pi^{*} \subset \Pi$. If $\theta$ is a Pfaffian on $M^{\prime}$, we let $\theta^{*}=\kappa^{*} \theta$. We can find bases

$$
\begin{aligned}
& \left(\omega_{1}, \ldots, \omega_{n} ; \widetilde{\omega}_{1}, \ldots, \widetilde{\omega}_{m}\right) \text { of }(\Omega, \Pi) \\
& \left(\theta_{1}^{*}, \ldots, \theta_{n^{\prime}}^{*} ; \xi_{1}, \ldots, \xi_{m^{\prime}}\right) \text { of }\left(\Omega^{*}, \Pi^{*}\right) \\
& \left(\theta_{1}, \ldots, \theta_{n^{\prime}} ; \eta_{1}, \ldots, \eta_{m^{\prime}}\right) \text { of }\left(\Omega^{\prime}, \Pi^{\prime}\right)
\end{aligned}
$$

so that $\eta_{\lambda}^{*}=\xi_{\lambda}-h_{\lambda}^{i} \theta_{i}^{*} ; \omega_{i}=\theta_{i}^{*}, 1 \leq i \leq n^{\prime} ; \quad \widetilde{\omega}_{\lambda}=\xi_{\lambda}, 1 \leq \lambda \leq m^{\prime}$, where the $h_{\lambda}^{i}$ are the usual functions on $M$ which we assume vanish at $p$. Let $a_{r}^{s \mu}$, respectively $\bar{a}_{i}^{j \lambda}$, be the structure constants of $\left(\omega_{r} ; \widetilde{\omega}_{\mu}\right)$, respectively $\left(\theta_{i}^{*}, \xi_{\lambda}\right)$ (and hence also of $\left.\left(\theta_{i} ; \eta_{\mu}\right)\right)$. As in Lemma 3.3 , we have

$$
\begin{aligned}
& a_{i}^{j \lambda}=\bar{a}_{i}^{j \lambda} \text { for } i, j=1, \ldots, n^{\prime} ; \lambda=1, \ldots, m^{\prime} \\
& a_{r}^{s \mu}=0 \text { for } r \leq n^{\prime} \text { and either } s>n^{\prime} \text { or } \mu>m^{\prime} .
\end{aligned}
$$

Suppose $u=\left(u_{1}, \ldots, u_{n}\right)$, respectively $v=\left(v_{1}, \ldots, v_{n}\right)$ is a vector in $\mathfrak{R}^{n^{\prime}}$, respectively $\mathfrak{R}^{n}$. Let $A(v)$ denote the $n \times m$ matrix $\left(a_{r}^{s \mu} v_{s}\right)$ and $\bar{A}(u)$ the $n^{\prime} \times m^{\prime}$ matrix $\left(\bar{a}_{i}^{j \lambda} u_{j}\right)$. If $v=\left(v_{1}, \ldots, v_{n}\right)$, let $\bar{v}=\left(v_{1}, \ldots, v_{n^{\prime}}\right)$. From (5) we find that

$$
A(\boldsymbol{v})=\left[\begin{array}{cc}
\bar{A}(\bar{v}) & 0 \\
? & ?
\end{array}\right]
$$


If we have vectors $u^{1}, \ldots, u^{q}$, respectively $v^{1}, \ldots, v^{q}$, in $\mathfrak{R}^{n^{\prime}}$, respectively $\mathfrak{R}^{n}$, we let

$$
\begin{aligned}
& \bar{A}\left(u^{1}, \ldots, u^{q}\right)=\left(\begin{array}{c}
\bar{A}\left(u^{1}\right) \\
\dot{ } \\
\dot{\cdot} \\
\bar{A}\left(u^{q}\right)
\end{array}\right) \\
& A\left(v^{1}, \ldots, v^{q}\right)=\left(\begin{array}{c}
A\left(v^{1}\right) \\
\cdot \\
\cdot \\
A\left(v^{q}\right)
\end{array}\right)
\end{aligned}
$$

We can find $n^{\prime}$ vectors in $\mathfrak{R}^{n^{\prime}}$ and a neighborhood of these vectors so that, for each $q=1, \ldots, n^{\prime}$, the rank of $\bar{A}\left(v^{1}, \ldots, u^{q}\right)$ in this neighborhood is maximal. Proceeding inductively and using (6), we can find vectors $v^{1}, \ldots, v^{n}$ in $\Re^{n}$ so that for each $q=1, \ldots, n$, the rank of $A\left(v^{1}, \ldots, v^{q}\right)$ is maximal and for each $q=1, \ldots, n^{\prime}$, the rank of $\bar{A}\left(\bar{v}^{1}, \ldots, \bar{v}^{q}\right)$ is also maximal. We fix these vectors.

Let $D=M \times M, D^{\prime}=M^{\prime} \times M^{\prime}, \rho=\kappa \times \kappa ;\left(D, D^{\prime}, \rho\right)$ is a fibered manifold. Associated to $\Omega$ and $\Omega^{\prime}$, there are the exterior differential system $(\Sigma, \Theta)$ and $\left(\Sigma^{\prime}, \Theta^{\prime}\right)$ on and $D^{\prime}$ respectively. Let $\Delta$, respectively $\Delta^{\prime}$, denote the diagonal of $M \times M$, respectively $M^{\prime} \times M^{\prime}$. Note that $\omega_{r}, \widetilde{\omega}_{\mu}, \omega_{r}-\Omega_{r}, \widetilde{\omega}_{\mu}-\Pi_{\mu}$, respectively $\theta_{i}, \eta_{\lambda}, \theta_{i}-\Theta_{i}, \eta_{\lambda}-H_{\lambda}$, are a basis of Pfaffians of $D$, respectively $D^{\prime}$, over functions and that $\omega_{r}, \widetilde{\omega}_{\mu}$, respectively $\theta_{i}, \eta_{\lambda}$, generate $\Theta$, respectively $\Theta^{\prime}$. We use a "partial derivative" notation to denote the tangent vector fields on $D$ and $D^{\prime}$ dual to these bases of Pfaffians.

Define tangent vectors $K^{1}, \ldots, K^{n^{\prime}}$ in $D^{\prime}$ at $p^{\prime} \times p^{\prime}$ and $L^{1}, \ldots, L^{n}$ in $D$ at $p \times p$ by

$$
\begin{aligned}
K^{i} & =v_{j}^{i} \frac{\partial}{\partial \theta_{j}} \text { summation from } j=1 \text { to } j=n^{\prime} \\
L^{+} & =v_{s}^{r} \frac{\partial}{\partial \omega_{s}} \text { summation from } s=1 \text { to } s=n
\end{aligned}
$$

where the $v^{r}=\left(v_{1}^{r}, \ldots, v_{n}^{r}\right)$ are the vectors chosen previously. Define contact elements at $p^{\prime} \times p^{\prime}$ by

$$
\begin{aligned}
& F^{i}=\left\{K^{1}, \ldots, K^{i}\right\}, 1 \leq i \leq n^{\prime} \\
& F^{n^{\prime}+\lambda}=\left\{\begin{array}{c}
\partial \\
\partial \theta_{1}
\end{array}, \ldots, \frac{\partial}{\partial \theta_{n}} \cdot \frac{\partial}{\partial \eta_{1}}, \ldots, \frac{\partial}{\partial \eta_{\lambda}}\right\} \text {, } \\
& 0 \leq \lambda \leq m^{\prime}
\end{aligned}
$$


The double definitions of $F^{n^{\prime}}$ and $E^{n}$ are consistent. Now it is clear that $E^{n+m}$, respectively $F^{n^{\prime}+m^{\prime}}$, is an integral element of $(\Sigma, \Theta)$, respectively $\left(\Sigma^{\prime}, \Theta^{\prime}\right)$, and is in fact just equal to the tangent space to $\Delta$, respectively $\Delta^{\prime}$, at $p \times p$, respectively $p^{\prime} \times p^{\prime}$. Since $\Omega$ and $\Omega^{\prime}$ are involutive, it is easy to check that

$$
\begin{aligned}
& \{p \times p\} \subset E^{1} \subset E^{2} \subset \cdots \subset E^{n+m} \\
& \left\{p^{\prime} \times p^{\prime}\right\} \subset F^{1} \subset F^{2} \subset \cdots \subset F^{n^{\prime}+m^{\prime}}
\end{aligned}
$$

are regular flags with respect to $(\Sigma, \Theta),\left(\Sigma^{\prime}, \Theta^{\prime}\right)$. Direct calculation shows that

$$
\begin{aligned}
& \rho_{*} E^{1}=F^{1}, \ldots, \rho_{*} E^{n^{\prime}}=F^{n^{\prime}} \\
& \rho_{*} E^{n^{\prime}+1}=\cdots=\rho_{*} E^{n}=F^{n^{\prime}} \\
& \rho_{*} E^{n+1}=F^{n^{\prime}+1}, \ldots, \rho_{*} E^{n+m^{\prime}}=F^{n^{\prime}+m^{\prime}} \\
& \rho_{*} E^{n+m^{\prime}+1}=\cdots=\rho_{*} E^{n+m}=F^{n^{\prime}+n^{\prime}} .
\end{aligned}
$$

We have $E^{n+m}=H\left(E^{n+m}\right)=\cdots=H\left(E^{n}\right)$ and likewise $F^{n^{\prime}+m^{\prime}}=H\left(F^{n^{\prime}+m^{\prime}}\right)=$ $\cdots H=\left(F^{n^{\prime}}\right)$. If $q \leq n-1$, then $H\left(E^{q}\right)$ is equal to the set of vectors $L$ at $p \times p$ of the form

$$
f_{r} \frac{\partial}{\partial \omega_{r}}+g_{\mu} \frac{\partial}{\partial \tilde{\omega}_{\mu}}+G_{\mu} \frac{\partial}{\partial\left(\tilde{\omega}_{\mu}-\Pi_{\mu}\right)}
$$

for which $a_{r}^{s_{\mu}} v_{s}^{t} G_{\mu}=0$ for $1 \leq r \leq n, 1 \leq t \leq q$. If $q \geq n^{\prime}$ and we apply $\rho_{*}$ to such an $L$, we obtain the vector

$$
f_{i} \frac{\partial}{\partial \theta_{i}}+g_{\lambda} \frac{\partial}{\partial \eta_{\lambda}}+G_{\lambda} \frac{\partial}{\partial\left(\eta_{\mu}-H_{\lambda}\right)}
$$

and by (5) we have $\bar{a}_{i}^{j_{\lambda}} v_{j}^{k}=0$ for $1 \leq i \leq n^{\prime}, 1 \leq k \leq n^{\prime}$. Since the number of linearly independent equations in this system is just $m^{\prime}$, we have $G_{1}=\cdots=$ $G_{m^{\prime}}=0$. It follows that $\rho_{*}\left(H\left(E^{q}\right)\right)=F^{n^{\prime}+m^{\prime}}$ for $q \geq n^{\prime}$. Similar arguments show us that corresponding to the sequences

$$
\begin{aligned}
E^{n+m}=H\left(E^{n+m}\right)= & \cdots=H\left(E^{n}\right) \subset H\left(E^{n-1}\right) \\
\subset & \cdots \subset H\left(E^{1}\right) \subset H(p \times p) \\
F^{n^{\prime}+m^{\prime}}=H\left(F^{n^{\prime}+m^{\prime}}\right) & =\cdots=H\left(F^{n^{\prime}}\right) \subset H\left(F^{n^{\prime}-1}\right) \\
& \subset \cdots \subset H\left(F^{1}\right) \subset H\left(p^{\prime} \times p^{\prime}\right)
\end{aligned}
$$

we have the relations

$$
\begin{aligned}
\rho_{*}\left(H\left(E^{n-1}\right)\right) & =\cdots=\rho_{*}\left(H\left(E^{n^{\prime}}\right)\right)=F^{n^{\prime}+m^{\prime}} \\
\rho_{*}\left(H\left(E^{n^{\prime}-1}\right)\right) & =H\left(F^{n^{\prime}-1}\right), \ldots, \rho_{*}\left(H\left(E^{1}\right)\right) \\
& =H\left(F^{1}\right), \rho_{*}(H(p \times p))=H\left(p^{\prime} \times p^{\prime}\right) .
\end{aligned}
$$


It is in order to be able to construct regular flags satisfying conditions (8) and (10) that we needed the assumption $S\left(\Omega, \Omega^{\prime}\right)=0$.

Now let $s_{1}, \ldots, s_{n+m}$, respectively $\bar{s}_{1}, \ldots, \bar{s}_{n^{\prime}+m^{\prime}}$, denote the system of characters of $\Sigma$, respectively $\Sigma^{\prime}$, at $E_{n+m}$, respectively $F_{n^{\prime}+m^{\prime}}$. Note that $s_{n+m}=$ $\cdots=s_{n+1}=0$ and $\bar{s}_{n^{\prime}+m^{\prime}}=\cdots=\bar{s}_{n^{\prime}+1}=0$. Let $\left(x_{1}, \ldots, x_{d^{\prime}}\right)$ be a coordinate system on $D^{\prime}$ compatible with the flag $(7)^{\prime}$; i.e. the origin of the coordinate system should be at $p^{\prime} \times p^{\prime}$ and

$$
\begin{gathered}
\left\{\frac{\partial}{\partial x_{1}}, \ldots, \frac{\partial}{\partial x_{r}}\right\} \text { should form a basis of } F^{r} \text { for } 1 \leq r \leq n^{\prime}+m^{\prime} \\
\left\{\frac{\partial}{\partial x_{1}}, \ldots, \frac{\partial}{\partial x_{h_{r}}}\right\} \begin{array}{c}
\text { should form a basis of } H\left(F^{r}\right) \text { for } \\
0 \leq r \leq n^{\prime}+m^{\prime}-1
\end{array}
\end{gathered}
$$

where $\bar{h}_{r}=\operatorname{dim} H\left(F^{r}\right)$. Because of (8) and (10), these coordinates can be raised to a coordinate system $\left(y_{1}, \ldots, y_{d}\right)$ on $D$ compatible with the flag (7). The correspondence between the two coordinates is of a rather complicated form which is perhaps best expressed diagramatically as

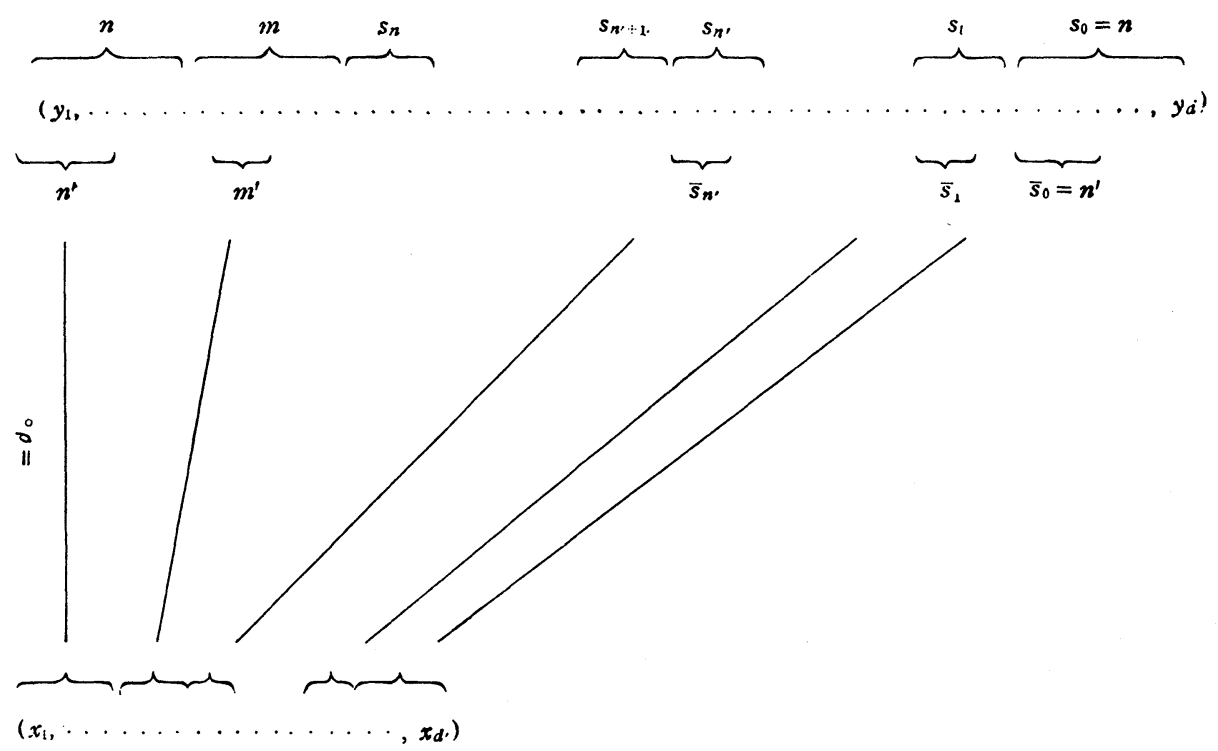

Lastly, we can assume that $\left(y_{1}, \ldots, y_{n+m}\right)$, respectively $\left(x_{1}, \ldots, x_{n^{\prime}+m^{\prime}}\right)$ come from coordinates on $M$, respectively $M^{\prime}$, which have been raised by $\alpha$, and which are fibering coordinates on $\left(M, M^{\prime}, \kappa\right)$. 
Let $\Phi$ denote a system of

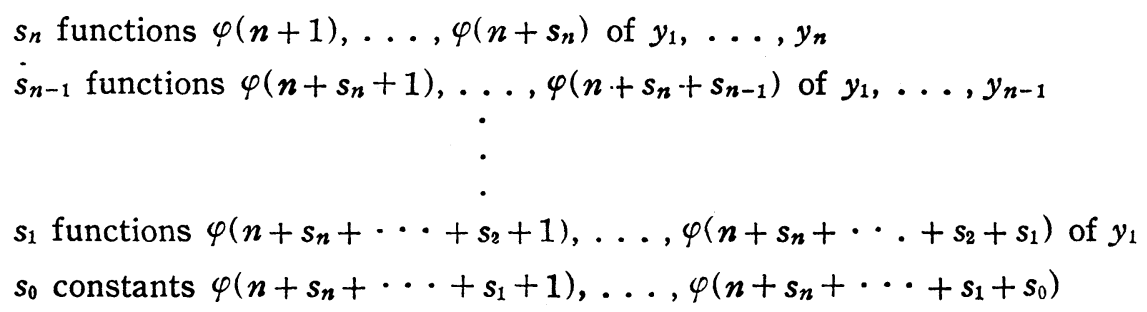

all these functions being defined in some neighborhood of the origin. By the norm of such a system, we mean the maximum of the absolute values of the functions $\varphi$ and their first partials at the origin. The Cartan-Kähler theory tells us that there is a one-one correspondence between systems $\Phi$ with small enough norm and solution manifolds of $(\Sigma, \Theta)$ which pass through a point of the form $p \times q$ in $M \times M$ and whose tangent space at $p \times q$ is sufficiently near $E^{n+m}$, i.e. in one-one correspondence with germs of transformations in $\Gamma(\Omega)$ defined at $p$ whose $l$-jet at $p$ is near enough to $I^{1}(p)$. This correspondence is "analytic" in a sense which can be made precise and has the property that if the system $\Phi$ corresponds to the transformation $f$, then

$$
(0, \ldots, 0, \varphi(n+1)(0, \ldots, 0), \ldots, \varphi(n+m)(0, \ldots, 0))=(p, f(p))
$$

Naturally, similar statements can be made for the system $\left(\Sigma^{\prime}, \Theta^{\prime}\right)$. Let $\Phi^{\prime}=\left\{\varphi^{\prime}\right\}$ be the unique system of fnnctions which corresponds to the solution manifold $\Delta^{\prime}$ of $\left.\Sigma^{\prime}, \Theta^{\prime}\right)$. Then it is clear that the systems $\Phi=\{\varphi\}$ which correspond to transformations in the kernel of

$$
(\Gamma(\Omega), M, p) \stackrel{\kappa}{\longrightarrow}\left(\Gamma^{\prime}\left(\Omega^{\prime}\right), M^{\prime}, p^{\prime}\right)
$$

are precisely those such that

$$
\varphi\left(n+m+s_{n}+\cdots+s_{n^{\prime}+1}+1\right), \ldots, \varphi\left(n+m+s_{n}+\cdots+s_{n^{\prime}+1}+\bar{s}_{n^{\prime}}\right)
$$

are equal respectively to $\varphi^{\prime}\left(n^{\prime}+m^{\prime}+1\right), \ldots, \varphi^{\prime}\left(n^{\prime}+m^{\prime}+\bar{s}_{n^{\prime}}\right)$

$$
\varphi\left(n+m+s_{n}+\cdots+s_{n^{\prime}}+1\right), \ldots, \varphi\left(n+m+s_{n}+\cdots+s_{n^{\prime}}+\bar{s}_{n^{\prime}+1}\right)
$$

are equal respectively to

$$
\begin{gathered}
\varphi^{\prime}\left(n^{\prime}+m^{\prime}+\bar{s}_{n^{\prime}}+1\right), \ldots, \varphi^{\prime}\left(n^{\prime}+m^{\prime}+\bar{s}_{n^{\prime}}+\bar{s}_{n^{\prime}-1}\right) \\
\vdots \\
\qquad \\
\varphi\left(n+m+s_{n}+\cdots+s_{1}+1\right), \ldots, \varphi\left(n+m+s_{n}+\cdots+s_{1}+\bar{s}_{0}\right)
\end{gathered}
$$


are equal respectively to

$$
\varphi^{\prime}\left(n^{\prime}+m^{\prime}+\bar{s}_{n^{\prime}}+\cdots+\bar{s}_{1}+1\right), \ldots, \varphi^{\prime}\left(n^{\prime}+m^{\prime}+\bar{s}_{n^{\prime}}+\cdots+\bar{s}_{1}+\bar{s}_{0}\right) .
$$

Under these circumstances, we say that $\Phi$ contains $\Phi^{\prime}$.

It is now easy to see that the orbit of $p$ under the action of $\operatorname{Ker}(\kappa)$ will just be the points $q \in M$ such that $\kappa(q)=\kappa(p)$, at least if we stay in some small enough neighborhood of $p$. Since this entire discussion can be carried out for any point in $M$, we see that $\left(M, M^{\prime}, \kappa\right)$ is actually the fibration of $M$ by orbits of $\operatorname{Ker}(\kappa)$. It is also easy to construct explicitly a transformation family in $\operatorname{Ker}(\kappa)$ which satisfies condition (2) of Definition 4.3 at $p$ by explicitly constru. cting the corresponding family of systems $\emptyset$. Again this argument works for any point in $M$.

Now let $G_{p}^{k}$ denote the manifold of $k$-jets of systems $\emptyset$ such that $\varphi(n+r)$ $(0, \ldots, 0)=0$. We have an obvious one-to-one bianalytic map $L: G_{p}^{k} \rightarrow J_{p}^{k}(M$, p) (the manifold of invertible $k$-jets on $M$ with source and target $p$ ) and there is a neighborhood $\mathfrak{u}_{p}^{k}$ of $I^{k}(p)$ so that the image of $L$ is $\mathfrak{u}_{p}^{k} \cap A_{p}^{k}(I, p)$. Restricting ourselves to $k$-jets of Goursat systems which contain $\emptyset_{0}^{\prime}$ determines a submanifold $\bar{G}$ of $G$ whose image by $L$ is just $\mathfrak{u}_{p}^{k} \cap A_{p}^{k}(\operatorname{Ker}(\kappa), p)$ which is therefore a connected submanifold of $\mathfrak{u}_{p}^{k}$. Again this argument works for any point in $M$. It is now easy to verify condition (3) of Definition 4.3 for $\operatorname{Ker}(\kappa)$.

Finally let $g$ be a homeomorphism element on $M$ and suppose there is a point $y$ in $U(g)$ such that $g(y)=y$ and $j_{y}^{k} g \in A_{y}^{k}(\operatorname{Ker}(\kappa), y)$ for all $k$. Certainly then there is an element in $\Gamma(\Omega)$ which coincides with $g$ in a neighborhood of $y$. Also $g$ in a neighborhood of $y$ is the prolongation of some element $f$ in $\Gamma\left(\Omega^{\prime}\right)$. Since $j_{y}^{k} g \in A_{y}^{k}(\operatorname{Ker}(\kappa), y)$ for all $k$, the partial derivaties of $f$ of all orders at $\kappa(y)$ must agree with the partials of the identity and so $f$ must itself be the identity in a neighborhood of $\kappa(y)$. Hence $g$ must agree with some member of $\operatorname{Ker}(\kappa)$ in a neighborhood of $y$. This shows that $\operatorname{Ker}(\kappa)$ is a continuous transformation pseudogroup and thus guarantees the existence of $\operatorname{Ker}(\varphi)$.

\section{REFERENCES}

[1] E. Cartan, Les systèmes différentiels extérieurs et leur application géométriques, Paris, 1945.

[2] E. Caratan, Oeuvres complètes, partie II, volume 2, Paris, 1953.

[3] V. Guillemin and S. Sternberg, "An algebraic model of transitive differential geometry", Bull. of the Amer. Math. Society, vol. 70, no. 1 (1964). 
[4] E. Kähler, “Einführing in die Theorie der Systeme von Differentialgleichungen”, Hamburger Mathematische Einzelschriften, vol. 16 (1934).

[5] A. Kumpera, Exterior differential systems, mimeographed notes, Princeton University, 1962.

[6] M. Kuranishi, “On E. Cartan's prolongation theorem of exterior differential systems”, Amer. Journal of Math., vol. 79, no. 1 (1957).

[ 7 ] M. Kuranishi, Lecture on exterior differential systems, Tata Institute of Fundamental Research, Bombay, 1962.

[8] M. Kuranishi, "On the local theory of continuous infinite pseudogroups, I", Nagoya Math. Journal, vol. 15, 225-260 (1959).

[ 9 ] M. Kuranishi, "On the local theory of continuous infinite pseudogroups, II", Nagoya Math. Journal, vol. 19, 55-91 (1961).

[10] M. Kuranishi, "The classification of complex simple continuous transitive infinite pseudogroups according to E. Cartan", mimeographed notes, Princeton University, 1961.

[11] M. Kuranishi and A. Rodrigues, "Quotients of pseudogroups by invariant fiberings", Nagoya Math. Journal. vol. 24, 109-128 (1964).

[12] A. Rodrigues, "The first and second fundamental theorems of Lie for Lie pseudogroups", Amer. Journal of Math., vol. 84 (1962).

[13] J. A. Schouten and W. v. d. Kulk, Pfaff's problem and its generalizations, Oxford, 1949.

Rutgers, The State University of New Jersey

New Brunswick, New Jersey 